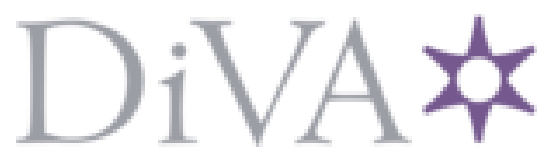

http://www.diva-portal.org

This is the published version of a paper published in Learning, Culture and Social Interaction.

Citation for the original published paper (version of record):

Samuelsson, R. (2018)

Children's explorations of the concept of spinning in preschool: Science learning in mediated activity

Learning, Culture and Social Interaction, 17: 90-102

https://doi.org/10.1016/j.lcsi.2018.01.002

Access to the published version may require subscription.

N.B. When citing this work, cite the original published paper.

Permanent link to this version:

http://urn.kb.se/resolve?urn=urn:nbn:se:sh:diva-34825 


\title{
Children's explorations of the concept of spinning in preschool: Science learning in mediated activity ${ }^{\text {th }}$
}

\author{
Robin Samuelsson \\ Södertörn University, School of Culture and Education, Alfred Nobels allé 7, 14189 Huddinge, Sweden.
}

\section{A R T I C L E I N F O}

\section{Keywords:}

Conceptual learning

Preschool

Perception

Artifact

Mediation

\begin{abstract}
A B S T R A C T
This paper examines how children explore the concept of spinning during a preschool project. It takes a cultural-historical approach, and analyzes how artifacts can be used in development of abstract concepts. In line with the pedagogical goals teachers employ these in learning activities during the project in line with their pedagogical goals. Children encounter the activities with different linguistic and perceptual means; there is, however, across the project a shift towards learning activities that promote verbal explanations. The interrelation of verbal and perceptual means, suggest ways in how children dynamically develop abstract concepts out of perceptual knowledge in activities with appropriate artifacts and teacher scaffolding.
\end{abstract}

\section{Introduction}

The aim of this paper is to examine how children explore abstract concepts in a preschool context during a project about the spinning motion. The paper analyzes different learning activities during the project, and how teachers employ artifacts to enable ways for children to explore the concept of spinning across the science project.

The pedagogical aim of the preschools' project was for the children to learn, which specifically includes verbal reasoning about spinning, all in accordance with the Swedish preschool curricula. The project emerged from the children's interest in the spinning tops used by the characters of an animated TV-series. These spinning tops, called Beyblades, were sold as toys for children to play, or "duel" with. Noticing the children's engagement and play activities, the teachers used spinning tops to initiate a project on "centrifugal force". Here, the teachers adapted the environment and staged both formal and informal activities in order to achieve science learning.

Vygotsky famously made the distinction between" spontaneous" or everyday and" scientific" or abstract concepts in the developing child, and the fundamental importance of cultural mediators. When elaborating upon this divide, Vygotsky (1986, p. 162) dynamically concluded that" we submit that the two concepts must differ in their development as well as in their functioning and that these two variants of the process of concept formation must influence each other's evolution.” This paper delves into this dynamic relation as preschool children (age 4-5) approach the spinning motion in the preschool setting, and more importantly, with a starting point in that children explore the concept mediated by linguistic as well as physical tools and artifacts.

In the cultural-historical psychological tradition, artifacts are seen as mediating between the individual and the environment and artifacts become "fundamental constituents of culture" (Cole, 1996, p. 144). In this tradition, material and intellectual tools, such as artifacts and concepts, are defining features of an institution, for example the preschool (Hedegaard, 2007). In the spinning project

\footnotetext{
Robin Samuelsson is a PhD student in applied linguistics at Södertörn University, Sweden. He is writing a thesis on how interaction in multimodal activities in preschool might afford learning and language development of children.

I have no acknowledgements to make.

E-mail address: robin.samuelsson@sh.se.
} 
studied specific artifacts and concepts are employed in accordance with the institutional settings and curricular goals. This is in line with developments in the cultural-historical tradition, also known as Cultural-historical activity theory (CHAT), that has added insights on how development occurs through collective activities, where knowledge is distributed among social actors and artifacts of the environment (Cole \& Engeström, 1993).

In this paper this is pinpointed in how children explore tools and artifacts that spins, such as spinning tops, during the preschool project. It specifically addresses how a sample of learning activities were mediating spinning artifacts are employed to promote ways for children to explore the concepts, and how the activities progress along the project. It is important to note that the preschool children explore their world and communicate their perceptions and understanding about it with different means - or as Finnegan (2014, p. 8) states, that humans "draw on a multitude of resources to interconnect with each other and in so doing interactively create their human world." In so, it also maps the relation between spontaneous and scientific concepts, in situ of the project activities, when children develop conceptual understanding with others around the artifacts of the preschool (cf. Vygotsky, 1986).

\section{Young children's science learning}

Preschool science in Sweden can be described as commonly functioning within the emergent science paradigm (Siraj-Blatchford, 2001), where children are actively encouraged to engage in exploration of natural phenomena for sustained periods of time together with peers and teachers. As such, play environments are key, as in these children can play with water, be outdoors in nature, and try out the properties of different artifacts with the guiding interactions of teachers and peers. Conezio and French (2002) consider preschool science activities to be heavily contextualized by the environment and artifacts as children experiment with them. The authors propose a connection between preschool science activities and language learning. Schoultz, Säljö and Wyndhamn (2001) point to artifacts as being central to children's reasoning about scientific concepts functioning as an auxiliary mean in children's reasoning about "scientific" concepts (cf. Vygotsky, 1986).

Sundberg et al. (2016) observe a large diversity among the science projects in three preschools. The different approaches to science in preschool can be traced back to the Swedish preschool model of educare, blending education and care. This possibly creates ambiguity in regards to science being a teacher-guided activity or based on children's own discovery learning. The authors find six categories of science activities, supported by the curricula: physical, sensory, creative/aesthetic, imaginative, play and storytelling. This study adds to this list how different types of activity might be used for science learning in preschool, and how different activities might be appropriated at different times during the project.

Important for this paper is how different means, including human perception, are used together with artifacts in science learning, something Goodwin (1995) pointed to as being central even for practicing scientists who use language, bodily means, the environment and artifacts to mediate their practices. Roth \& Lawless (2002, p. 380) draw parallels to scientists learning through deictic and iconic gesturing to science students during a science project in school. The students followed a pattern of going from manipulation and feeling of objects, to symbolic gesturing, and later emerging into descriptive and theoretical language. The authors go on to conclude that "hands-on learning affords new forms of observational and theoretical talk." For this paper, this sequence of learning is to be noted, and the learning activities studied will show how language and gestures, along with other means progresses over time during the observed project.

Such other means of explorative activities have been studied by Klaar \& Öhman (2014) who point to the centrality of actions and bodily movement when children engage in nature, and how teacher actions may promote children's possibilities to further engagement in nature-oriented preschool activities. They highlight the interactions between individual and environment, in both teacher-planned and spontaneous activities, and how teacher instruction and confirmation of children's movement can guide learning in outdoor activities, sometimes blurring the distinction between education and care. This emphasizes how science learning in Swedish preschools is not content-based, but rather multifaceted and relies heavily on the child's experiences of everyday phenomena. Larsson (2013) studied four children's exploration of friction during their days at a preschool. The children explored friction in everyday activities, sometimes in disjunction with the institutional agenda. Larsson (2013) observed critical moments when children played in the preschool environment and used its artifacts, such as when friction increases when a sledge gets stuck, creating an opportunity for reflection about the phenomena. However, she observed that such opportunities often fail to be observed by teachers.

The dual nature of the preschool as both supplying care and explorative activities where children can learn, is manifested in different ways, as mentioned. Sundberg et al. (2016) studied a preschool conducting a rolling and spinning project. The children were supplied with artifacts such as beads, balls and cylinders that they used to explore rolling and spinning. Besides this, they did exploration during physical activities such as rolling down a slope. However, the children only explored these motions themselves without any guidance of teachers, as their pedagogical goal was to merely provide the children with the opportunity to discover the phenomena for themselves. This study will add to how such tensions, emanating from the different goals, or objects of children and teachers, might be productive during one and the same science project.

For this study, ways in which children explore concepts through mediating tools and artifacts are of fundamental concern. Pramling \& Pramling Samuelsson (2001) takes their starting point in that scientific thinking of preschool children must be based on the fundamental understanding that phenomena can be explained. They follow a young child (3.3 years) who experiences and tries to understand the concepts of floating and sinking, and actively explores these concepts with teachers and in play. The play with different artifacts in water is seen as a sort of hypothesis testing. The teacher makes the child come up with predictions that are tested in play. The authors conclude that children develop their spontaneous concepts in experiencing, thinking and talking about them with others, long before the concepts are fully grasped by the child. 
This study sets out to contribute to research on how such activities can be seen as learning activities and how these fit into the larger frame of a preschool project, where they develop these upon previous common experiences of the children. Following the plea of Thulin \& Pramling (2009, p. 139) who state that" empirical research on science learning in preschool settings are rare", this study addresses this under researched area. It adds understanding about the means that children use in exploration of a concept and how this unfolds in activities mediated tools and artifacts during a preschool project.

\section{Theoretical approach}

In the cultural-historical approach to child development, development and learning relies on interaction with others through mediating tools and artifacts in human practices and activities (Cole, 1996). In the CHAT tradition, activities are seen to take place in an Activity System, where the activity mediated by tools or artifacts also is emplaced in the community and its settings with its applied rules and divisions of labor. Such systems are seen in relation the object of the activity under analysis. Mediated events are acted out in line with the object of activity (Sannino, Daniels \& Gutiérrez, 2009). This study does not fully use analyze the preschool as an activity system as formulated by Engeström (1987), it however uses important concepts from this tradition, and does have affinity with CHAT in that it studies the tensions and overlaps of the goals, or objects, of children and teachers. Especially how the mediating artifact is central for these overlapping interactions to function, where participants in the same setting are sometimes acting according to different activity systems, with different objects (Cole \& Engeström, 1993). This is an important insight for this paper, since the object of children's play and the object of teachers and curricular goals may diverge in the very same activity.

It should be understood how the learning activities are emplaced within a Swedish preschool and how the object of the activity is related to those curricular goals of the institution. Hedegaard (2007) theorizes how the preschool is a socioculturally shaped institution contained with concepts, artifacts and tools. These are related to the preschools goals and interactions among teachers and children inside the preschool can be analyzed in light of this. The paper therefore adheres to many of the central tenets of CHAT, in that human thinking and learning is distributed across minds, artifacts, activities and setting (cf. Cole \& Engeström, 1993; Cole 1996).

The preschools learning activities studied should be seen as arranged in this manner, not only though direct instruction, but also that teachers may arrange the preschool environment, its tools and artifacts, and that these physical setting can be seen in relation to the preschools goals (cf. Hedegaard, 2007). Nelson (2007) propose that children from a very young age can derive a conceptual understanding through acting, both verbally and physically, in the events of socioculturally arranged settings.

As Wartofsky (1979) pointed out, perception is not immediately direct, and can be seen as part of a mediated activity. This aligns with the multimodal approach to mediation of this study. Following this, human movement can be seen both as a vehicle to sense and explore and act within the setting (Ingold, 2000). By touching, feeling and experiencing the material world, and interacting with it in social ways with others, children come to actively explore, enact and change their settings (Streeck, 2009). Artifacts are guiding in these interactions and are "simultaneously ideal and material. They coordinate human beings with the world and one another in a way that combines the properties of tools and symbols" (Cole, 1996, p. 144). Children can in this way derive understanding from their perceptual exploration of the material world around them (cf. Wartofsky, 1979), and artifacts as such can work as a scaffold for emerging understanding of phenomena. When children talk about their experiences in interaction with more experienced interlocutors, knowledge about these phenomena might be developed, as the dynamics of everyday and scientific conceptual intermingling unfolds in activities (cf. Vygotsky, 1986).

Central to these exploratory activities is the use of the human hand(s). The human hand is used as a both inter- and intrapsychological resource and can be used as a mediational tool (Vygotsky, 1978). The hands work as a mean for communication as much as a tool for feeling the immediate material world and gather information about its properties. Gestures, are an important mode for communication (Finnegan, 2014; Streeck, 2009) and bodily expression can be perceived by teachers as cues to scaffold learning (Goldin-Meadow, 2015), creating a zone of proximal development (Vygotsky, 1978). McNeill (1992:86) describes the "gesture space", that is the proximal area where pointing at and touching the immediate surrounding is possible. When categorizing types of gestures, McNeill (1992) described iconic gestures as either a pictorial use of hands in describing a content or the closely related category of metaphoric gesture that is used to depict abstractions. Deictic gestures are variants of pointing, commonly used by children and interlocutors to direct others to physically proximal artifacts. However, pointing can indeed also refer to a virtual or imaginary space. Yet, it is important to note that all means, including other bodily means, perception and language, complement each other (Streeck, 2009) and they are seen as "environmentally coupled" (Goodwin, 2007) with the surrounding, including its artifacts, in communication and learning (c.f. Finnegan, 2014).

The learning activities of the science project studied should be seen in light of the emerging conceptual development of the children, and the multimodal acts as part of the development towards verbally mediated abstract concepts. These activities are emplaced within the preschools overarching institutional goals (cf. Hedegaard, 2007), and should for this study be seen as examples of how children development of abstract, 'scientific' abstract concepts in a dynamical relation with their everyday understandings that in ways are perceptually grounded.

\section{Methods}

Knoblauch (2005) describes a focused ethnography as the intense collection of data in a briefer period of time compared to the classic anthropological fieldwork. Using audiovisual recordings, together with other techniques of data collection, a large amount of data can be collected in relatively short periods of time.

A visual approach to data collection and analysis has been employed for this project (cf. Pink, 2015). This aligns with a cultural- 
historical approach where the environments or activity systems (cf. Cole, 1996) artifacts, here especially the spinning-top toy is used for its mediating potentials as the children move towards explanatory, 'scientific', language throughout the project. The data for this project were collected via: video observations of instructional, teacher-guided, and play activities with synchronized field notes; photography of settings and other artifacts such as toys; informal interviews with teachers and children; and documentation of the preschool project made by teachers.

In the corpus, the word centrifugal only appears once, during a PowerPoint made for parents and educational leaders in the municipality. Henceforth, the word 'spinning', more commonly used by both the teachers and children, will be used.

The unit of analysis is the 7-week project about spinning and captures the children in learning activities related to this. All recordings were initially coded for any events that could be connected to spinning, both when referenced linguistically or by the children's actions. Due to the popularity of the spinning tops among the children, the spinning motion was often explored via a range of means. The variety of activities that was coded covered all of the six science activity categories of Sundberg et al. (2016) throughout the project. The sampled data for this paper focus on activities in which children try to understand and express themselves about the spinning motions. It was clear that the use of bodily movement, touch, language and gestures among a multitude of means was employed by children when exploring and expressing themselves on the spinning motion, as this was seen throughout the corpus.

The children, in this sense, explored the concept multimodally, and an analytic point of entry is to establish how different modal configurations of mediational means (Norris, 2014) are arranged by the teachers. These are termed 'learning activities', and four of these will be analyzed in the paper. The learning activities are defined as situations that are configured within the spinning project with a goal of children learning about spinning, either in activities set up and scaffolded by the teachers or in the children's participation in settings that enables exploration of spinning through mediated activity.

It will be evident that there is a dynamical relation between a pre-verbal sensing and explicit verbal reasoning, as different activities promote types of modal configurations (Norris, 2014) to be used, these should however be seen in light of the overarching object of the activities (cf. Sannino, Daniels \& Gutiérrez, 2009) as guided by the preschools curricular science goals.

\section{Analysis}

The analysis begins with a contextualization of the commercial spinning top followed by an illustration and analysis of how it is appropriated into the preschools' learning activities (see Diagram 1) during the project. The activities are defined by the teacher's pedagogical values and goals in interaction with the children's interest, and is materialized in the preschools environment in settings, artifacts and employed as learning activities. Then follows an analysis of the four learning activities: construction, competition, bodily movement and reasoning. Learning activities, (LA) should be seen as part of the preschool as a cultural-historically shaped institution and activities will be presented chronologically in line with how the project progressed. The situations are somewhat comparable to the progression that Roth \& Lawless (2002) saw in school children, where the students went from physical touch to gesturing to verbal and theoretical language. In this case, language together with perceptual means are present throughout, however, there is a movement towards configurations foregrounding verbal language use in the later stages of the project.

\section{The learning activities}

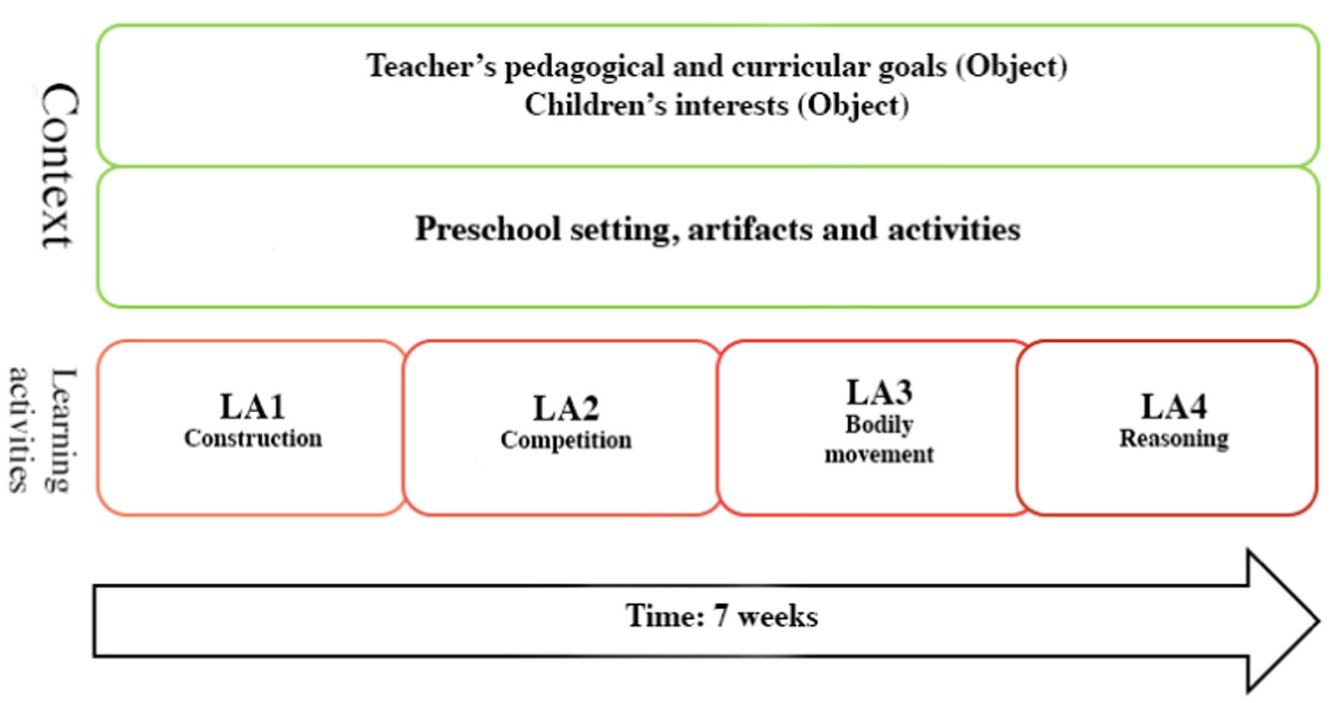

Diagram 1. The preschool's learning activities mapped along the 7-week unit. 
With a cultural-historical approach, the environment of the preschool can be seen as dynamically changing during the project as in line with the changing goals, or object, of activities (cf. Cole \& Engeström, 1993). It should however be noted, that these activities are set in the institution of the preschool with an object that resonates with the Swedish preschool science curricula (cf. Hedegaard, 2007). The institutional goals, or object, is sometimes diverged with the goals of the children, that might use the spinning-top as a leisure activity. The tensions should be noted, but also as potentials of how children's object of activities, i.e. goals or interests, can be overlapping with activities with pedagogical goals. In LA1 it is highlighted how such change to the preschool's activity system offers new ways of solitary engagement and construction of spinning artifacts: The trial-and-error type of construction interestingly would not have been possible with the commercial toy that comes out of the box without possibilities of add-ons. In LA2 the focus is on how the constructions are used among peers when competing though spinning top duels.

During the project, activities are arranged for the children to explore spinning in different ways, all within the pedagogical goals of the project. In LA3 it is analyzed how children's own spinning movements are acknowledged and remodeled to a pedagogical activity by adding a bucket of water. In LA4, we look at the reasoning of the children through a number of means when recalling and reflecting on LA3.

\subsection{Context: Spinning tops in the preschool's learning activities}

Before the preschool project that was adopted by the teachers, the children had shown a growing interest in the commercialized Beyblade-concept, through a Japanese animated TV-series and comic or "manga". The main artifacts in the narrative are spinning tops that different characters use to contest and duel each other. Children replicate the animated series by spinning their spinning tops against each other with the goal of stopping the opponents' toy.

The toys (left in Picture 1) comes prefabricated, in a one-size-only, with merely color and some small extensions differentiating the items. The different Beyblades are used by certain characters in the series. Subsequently, children identify with characters, and it makes them into popular collectibles.

Inspired by the pedagogical philosophy of Reggio Emilia which focuses on the creative abilities of children, the preschool has implemented rules against toys being brought in to the preschool. At the same time, they adhere to a child-centered approach where children's experiences, interests and life-worlds are an important basis for children's own involvement in their learning process. Following this, the commercial concept was appropriated by the teachers to make it more pedagogically sound, all the while still preserving the children's interest. This was the main reason for the teacher's creation of the preschool project called "Beyblades and the centrifugal force"; in that it can be seen as a form of emergent science project clearly grounded in the children's interest in the spinning top artifact. As previously mentioned, the settings and activities were changed, added on and arranged during the project. One important activity during the project was the "trial-and-error" construction of spinning tops (its popularity is shown in Picture 2), this can be seen as a form of trying out artifacts' properties comparable to the ones observed by Pramling \& Pramling Samuelsson (2001).

As the teachers observed a need, more materials could be added. Such changes do not only allow for more peers to construct the artifacts together (as in Picture 2), but also enables new kinds of constructions to be made (see picture 3 for examples of the materials

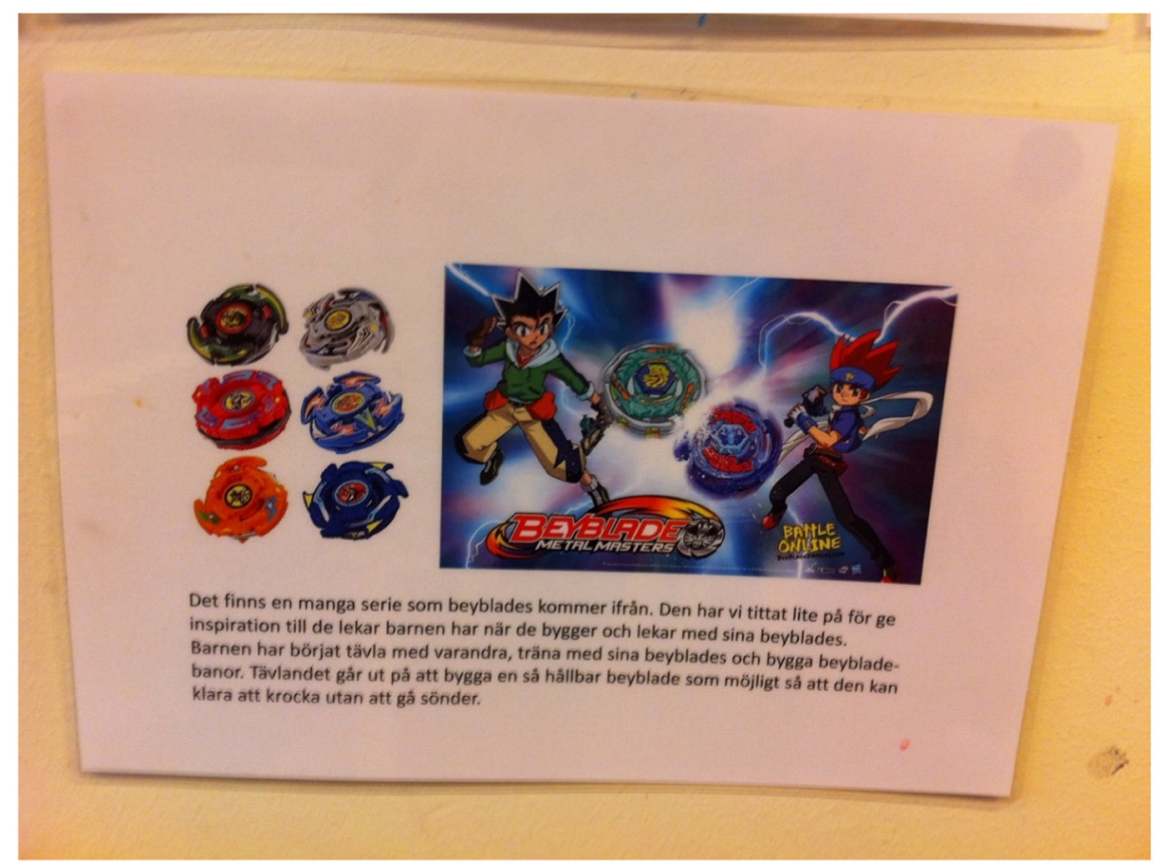

Picture 1. The Beyblade concept presented in a project presentation for parents at the preschools' lunchroom wall. 


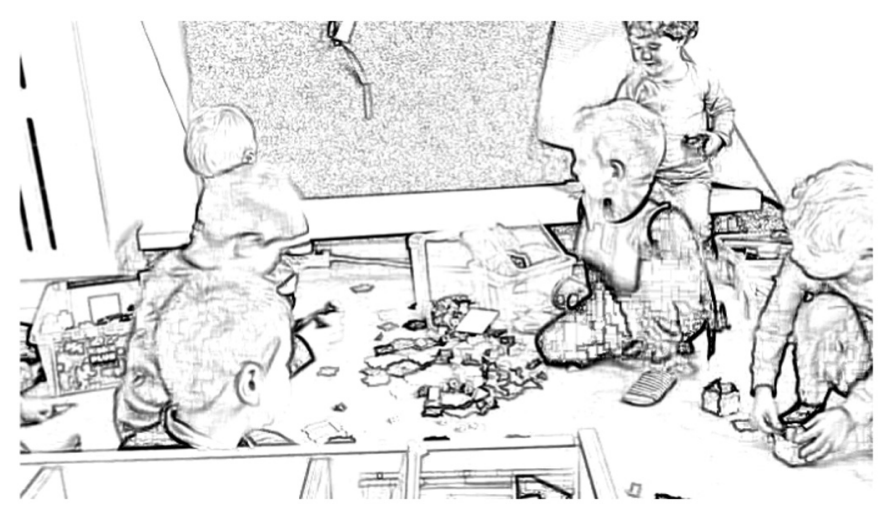

Picture 2. Several children (6 in picture, 2 out of frame) assembling spinning tops out of different construction materials.

and constructions). We will now turn to what such construction can enable in the analysis of LA1, and what means children can use when doing this.

\subsection{Learning activity 1: construction}

In this learning activity we look at what one of the construction materials supplied by the preschool can enable in regards to children's understanding of spinning. As previously mentioned, teachers added new construction material during the project, and the amount of material can here enable for the child to explore certain properties of the spinning-top artifact.

The learning activity illustrates how a boy uses the perceptual means of touch, gaze, and listening when constructing a spinning top, and by these perceives the properties of his constructed artifact. Throughout his demonstration, he talks and makes remarks and reflections about what he is doing, drawing the focus to certain important steps when assembling, such as that pieces should "click" together to stick. This shows how language is coupled with other means as children build their understanding. These type of means mediate his experiences and may later be used when competing, understanding and later reasoning about spinning, with language being the central tool to abstract knowledge about the world (cf. Cole, 1996).

Picture 4 shows the boy constructing a spinning top. As he does this, he displays it to the researcher and makes deictic expressions as he assembles it, such as "look at my beyblade!", drawing the attention towards the artifact being constructed. This scene is for analytical purposes divided by perceptual means and it exemplifies the different senses the children make use of in the construction of spinning tops. The child of our example explores the properties by testing the artifact, something that can be compared to Pramling \& Pramling Samuelssons (2001) study of a child's explorations, and also their sequence of experiencing-thinking-talking can be noted here. The children use their hands to touch the material artifact, to perceive its properties, by gazing at a spinning object whereby the attuned eye can make inferences of its functioning. By listening to the spinning sound, the children may further attend to any irregularities in how the spinning tops perform. These sensory categories are used to illustrate the use of the perceptual means when children engage in activities and how they are coupled with verbal language throughout the learning activity.

\subsubsection{Touching and feeling}

By touching the construction, children may get a feel for the properties of the artifact at hand. In this example, the boy is shown when perceiving the overall balance of the spinning top he is constructing. This is explicated by his words that "this one helps to spin in the middle". In this construction, the middle is key for a balanced spinning, and this has been noticed by the boy. The symmetrical shape is more crucial in a relatively small construction like this. The artifacts properties may be perceptually explored by touching the artifact while constructing - feeling its weight, shape, and eventual imbalances.

\subsubsection{Gazing and attending}

By gazing at artifacts, either in solitude, or jointly with others, children may attend to what they see, and by the perceived properties of what they see, they may act upon it. Here, the boy in the example is testing his spinning top in action, "look how it spins", the boy calls to the camera. While it spins, the boy locks his gaze on the spinning top, attending to it. When it slows down, he distinctly grabs the spinning top and continues to assemble more pieces onto it.

This exemplifies the common trial-and-error behavior of the children when constructing artifacts. The construction material enables new pieces to be assembled onto an existing construction (compare with Lego). This property is frequently tried out by the children and supported by the preschool teachers and their pedagogical goals, as more pieces are added to the playroom when the popularity of the project was acknowledged. As such, it enables the children to explore the spinning motion of spinning tops of different sizes and weights.

\subsubsection{Listening}

When the boy continues to construct the spinning top, more means are exploited. As he continues to add pieces, he displays his constructed artifact and tells the researcher that "this is how you build really big beyblades". As a final extension of the spinning top, 


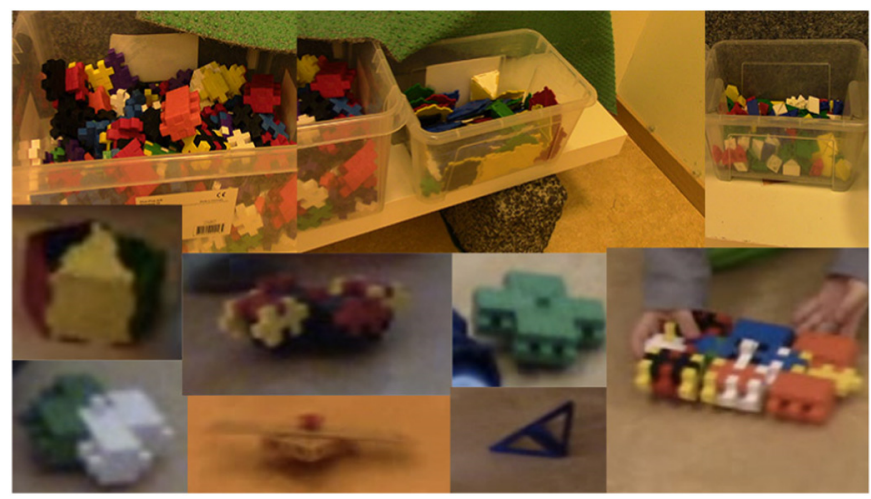

Picture 3. An assemblage of pictures displaying different construction materials and an array of different constructions made by the children.

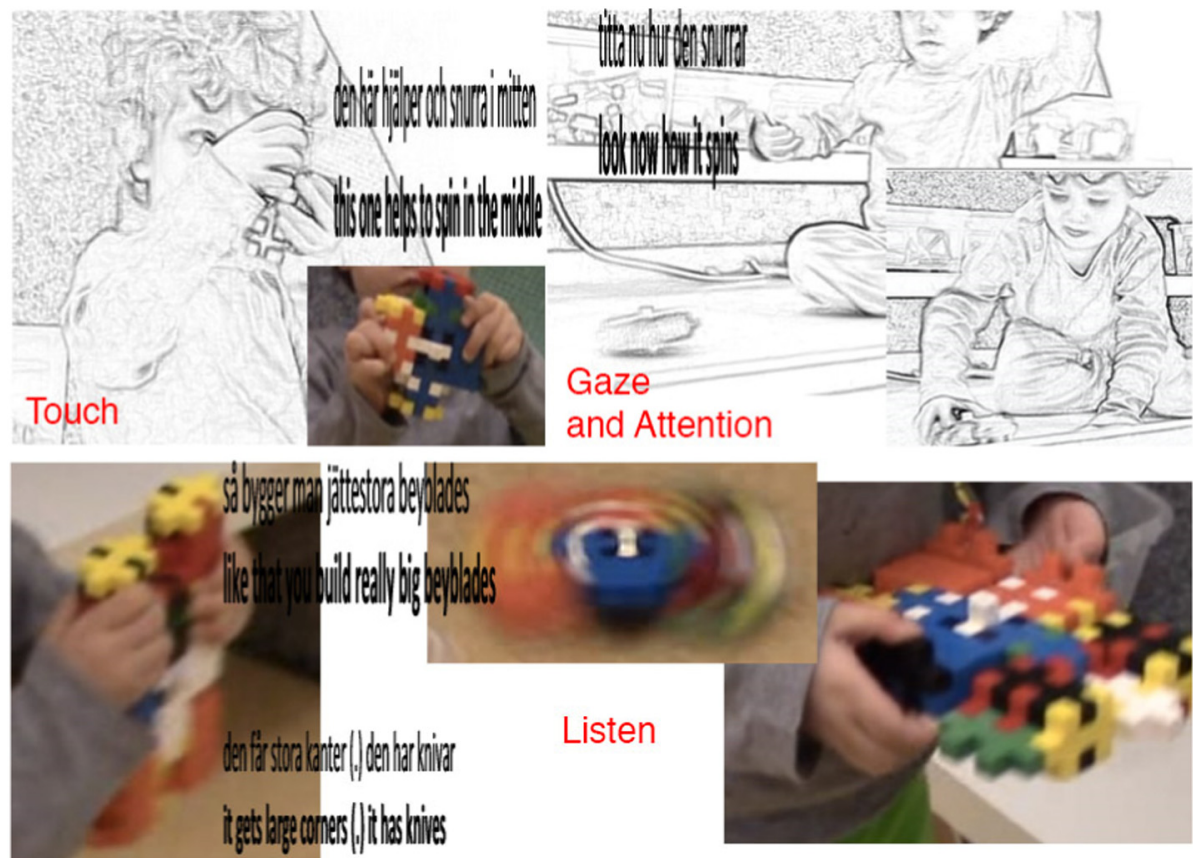

Picture 4. A boy constructing a spinning top with the use of language and different sensory means.

he unfolds its side pieces, naming them firstly "corners" then "knives" (as they are depicted in the animated movies and toys). When showing this construction in action, while attending to it as it spins - a clunking sound is heard as the spinning top starts to wobble. This seems to be registered by the child, because he grabs it to fold the outer pieces back in, then re-spins this construction without neither the wobbling nor noise, now with the middle as a balanced center as he before explicitly noted. Through listening and attending to this larger and uneven construction, it enables the child to explore how spinning artifacts might be affected by symmetrical imbalances. Like in Larsson's (2013) observations, this can be seen as a critical moment that arises when artifacts are not working as children expect them to, promoting possible reflection.

\subsubsection{The use of multiple means of perception}

The array of means, including the sensory perceptions of children (here exemplified from one recorded event) show how children are interacting with the material world and its artifacts properties through different senses, coupled with language as children initially form understanding of phenomena. By feeling with their hands, getting a sense of spinning patterns, carefully attending, and using other means such as hearing, the material setting of the preschool learning activities enables the children to explore the spinning object through different perceptual means and talk about what they perceive and understand. By putting words to what they are perceiving, children can categorize their experiences, and as we will examine later, interrelate with others towards common understanding of the world around them. This learning activity is in line with the free-spirited activities, referred to as "free play", in Swedish preschools, where children can explore and play in their settings with a high degree of freedom. In LA2, it is shown how this 
is done socially among peers, where the constructed spinning tops are tested for their durability when children compete with their spinning tops against each other's' created artifacts.

\subsection{Learning activity 2: competition}

This section displays an example of how play dueling during the project can enable the children to use basic talk, gesturing, and sound-making (clapping) when interacting around the spinning tops. Exploration, is not only a solitary matter as in LA1, it is also a pedagogical idea often promoted in Swedish preschools, in that children should explore artifacts together among their peers, consistent with an emerging science knowledge (cf. Siraj-Blatchford, 2001).

The following Excerpt 1 is a scene where four children are sitting around the "arena" - an upside-down table without legs, arranged by the teachers for the children to use in their spinning top duels. The idea of the arena comes from the commercial Beyblade toy. Here, it enables the children to interact when competing against each other, around the arena. Foregoing this learning activity is the children constructing their respective spinning tops. Three children are actively engaged in the duel, and a fourth is sitting by and constructing a spinning top in preparation for an upcoming duel.

Two children at opposing sides are dueling each other, and the third is here acting as a referee. He is counting down with formulaic expressions to start them off: "three two one" and "get started!". Sometimes while they are dueling, one spinning top may stop the other, forcefully, so that pieces fall off. In this case, there is no such clear winner. The spinning tops barely touch, both slow down, with one decelerating to a stop right before the other. This causes ambiguity, as the first child asks "who won?" to the referee. The other child questioningly asks "but were both not crushed?". The referee wants to tell that the first child won the duel, but this time without any violent crash. This is done by using the clapping - "yeah but not like boom" as he claps his hands together.

In this example, where the children are dueling, they can also be said to be exploring spinning and related concepts of power, force and speed on an intuitive level. However, these are enacted with an emphasis on bodily means within their play, and never thoroughly developed upon with elaborated verbal language. Instead, formulaic language and words like "crushed", "boom" and clapping are used to discuss the spinning tops' soft collision.

In the learning activity, gesturing is used to complement talk and the interaction is reliant on the objects and the sound they produce (or in this case the absence of the sound 'boom'). Thus, verbal explanations are backgrounded in this configured setting. At the same time, the children seem to display a tacit understanding of how the spinning tops may function, as they solve the negotiation through a joint agreement with relatively little verbal dialogue. As such, they interconnect in this joint activity in a sufficient way for their duel to take place with low verbal interaction (c.f. Finnegan, 2014), as this is sufficient to accomplish the children's goal of the dueling activity. It may be argued that this activity do not do anything to develop the children's knowledge. However, it will later be presented in LA4 how bodily means of expression are used when children try to discuss higher order concepts with their teacher. LA3 analyzes a different way in which the body is used as a mean in children's exploration of spinning and how this can be used to make hypotheses about the world around them.

\subsection{Learning activity 3: bodily movement}

Throughout the project, children are seen spinning themselves in different ways. Spinning, of course, can be seen as a normal way of children playing with the weight of their bodies in the space around them. However, the spinning is frequent and it is clear that this is sometimes inspired by the Beyblade project at the preschool, as children spin themselves in directly related activities, such as when dueling. In play, children may explore the properties of objects situated in the environment of the preschool; here they themselves act as Beyblades, allowing the body to work as a tool mediating the phenomena by their own acting.

In Picture 5, the children have progressed from a dueling inside the arena to they themselves acting as Beyblades inside the very same space; "we are monster-beyblades" announces one child. They spin carefully, not to hit each other's arms - announcing that "we are friends". When exploring together (as seen a variant of in LA2) children do not only have to explore the material surrounding, but

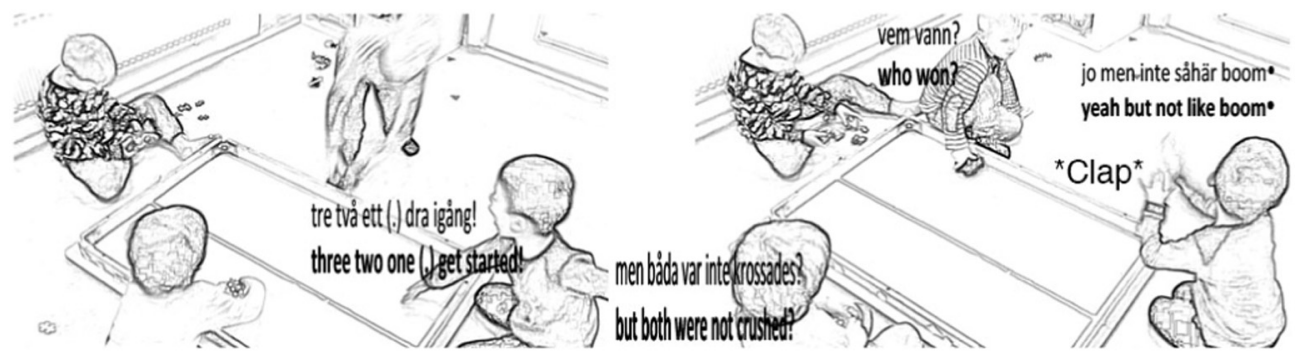

Excerpt 1. Children competing with their constructed spinning tops inside the arena. 
also the relation with each other. As before, language is used to name experiences and feelings, and here it follows a social function, as it concerns interrelations with peers. Contrasted with the example of dueling, with spinning tops violently crashing, this puts the experience of spinning into another perspective. Here, children are sensing spinning themselves, as another way of perceiving the phenomena, thus feeling the weight and force of their bodies while rotating.

The interest and enthusiasm surrounding the spinning motion is acknowledged by the teachers and this inspires the teachers to arrange new activities, such as the one seen in Picture 6 where a boy is seen spinning while holding a bucket containing some water. Referring to the understanding of centrifugal force as the goal of the project, the teachers here want to enable the children to experience that spinning themselves with the bucket rapidly enough ensures that water will stay inside the rotating bucket.

This can be labeled as an emergent science activity created by the teachers. However, it is an learning activity where both the perception of the world by children through exploratory play and the preschools pedagogical goals converge. As this happens, the buckets provided by the teachers add a tool to the children's experiential world for perception of weight and the force of water against the inside of the bucket while spinning. Therefore, this adds a way for first-hand knowledge on the concept of spinning through their whole body as mediating the experience. During the activity, they can make up hypotheses, in situ, together with the teachers (c.f. Pramling \& Pramling Samuelsson, 2001), such as the one that water is "pushed back" into the bucket - once again coupling language and the children's perceptions in the forming of emergent understanding of phenomena.

In the next section, we see how children later on elaborate and use a range of means when reflecting back on this activity. The children will be shown to use their array of sensory means, coupled with other linguistic and environmental tools in their explanations and arguments about spinning.

\subsection{Learning activity 4: reasoning}

This example consists of a close analysis of three children looking at photos of the activity described in LA3 together with their teacher. She is showing a projection of children spinning their buckets on the wall. The photos projected are of the outdoors activity in Picture 6 when children spun themselves while holding buckets of water in order to try out if they could spin it without water pouring out Excerpt 2.

The dialogic interaction sustained by the teacher promotes the use of verbal language as a more prominent mean in this learning activity. The interaction in this situation puts an emphasis on the use of verbal language, it is sometimes reminiscent of the IREstructure of direct instruction, where a question from the teacher demands a verbal response from the interacting child to be evaluated. The explanations can be considered as relatively advanced reasoning for these children. The example will also point out to how their reasoning is combined with different types of gesturing (deictic, iconical, and metaphorical), other bodily means, and at times done in interaction with the environment, such as when the virtual interface is deictically used as a tool for explanation.

The reasoning illustrates how the children's explanations couples spoken language and bodily means. In addition, with the digital projection being the center piece, the artifact is at times integrated with the children's expressions, highlighting the role of environmental coupling of artifacts in children's reasoning (c.f. Schoultz, Säljö \& Wyndhamn, 2001) as the children use them deictically. This is of course a variation of the fundamental question for Vygotsky (1978), of what auxiliary means allow children to intellectually do. The projected image in this learning activity, is an artifact containing a recollection of a past activity. Hence, this learning activity in several ways promotes reasoning, where verbal language is an integral and central mean in abstracting from the physical world (cf. Cole, 1996).

The focal children are here called (from the left in Excerpt 2): Lucas, Axel and Elias. The teacher is standing left of the camera,

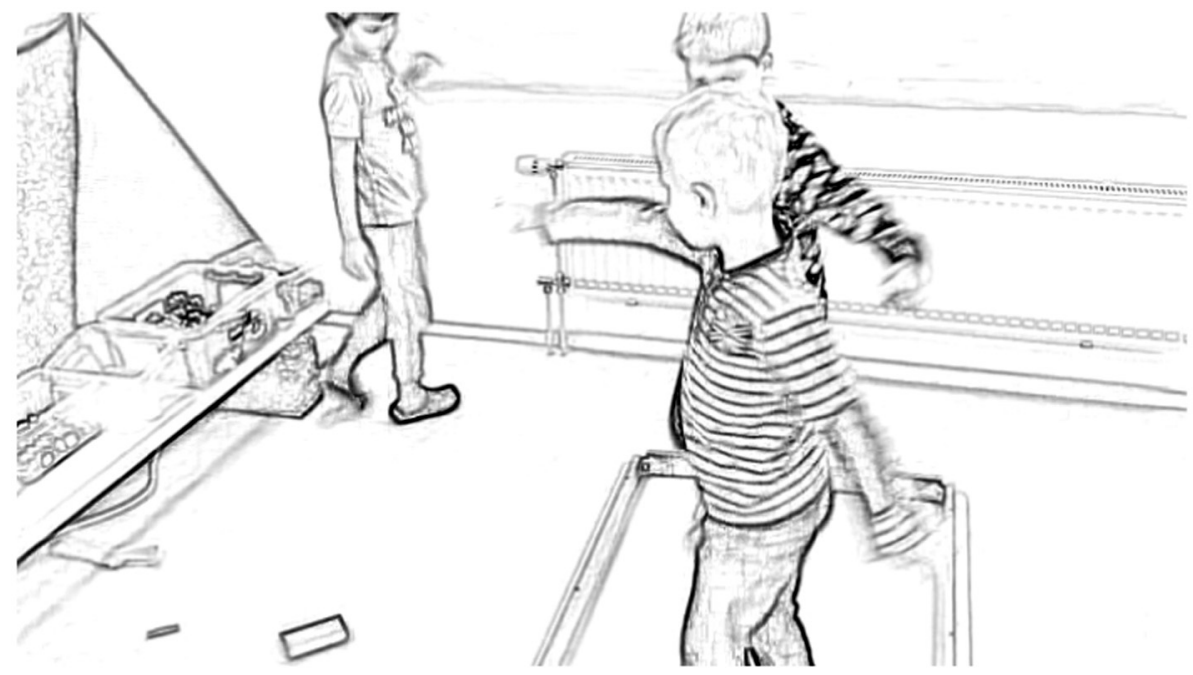

Picture 5. Children roleplaying Beyblades inside the arena. 


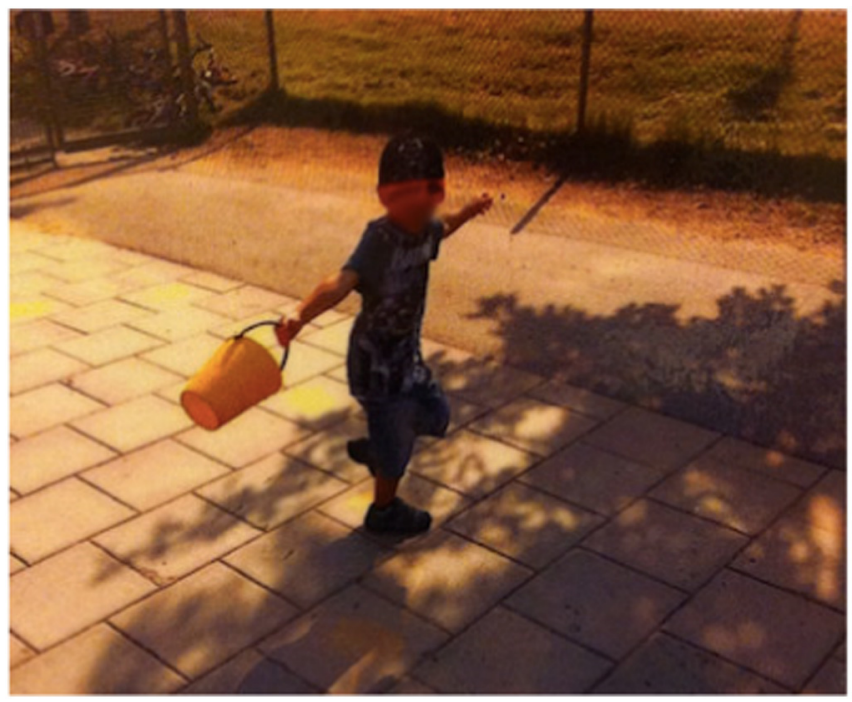

Picture 6. A child spinning with a bucket containing water.

behind the children, while she is controlling the computer connected to the projector.

The example begins from the teachers' seemingly routine question to the new slide of "what's it you're doing here then Axel?" (Line 1). Axel addresses the deictically available screen and points towards it, and follows with the answer that "it's water then". This sentence is subsequently completed by a relatively large sweeping motion with the hand over the visible field (line 2). The teacher follows with another routine, or instructional-like question about the containers (line 3). This question is quickly replied to by Axel and Elias with "bucket/s" (line 4). Elias then continues to tell that you spin it (line 5) and Axel continues to elaborate on that by saying "it stays put" (line 6). Elias reflects on this and questions Axel's statement "does the water run out when you spin it?" This question is emboldened by the use of iconic or metaphorical gestures, first as he says "out" he points his index finger and drops it towards the ground. He then moves his hand up and spins it in the air as he says "spins it" (line 7). Axel stands by his argument by saying "no then it stays put", the word "put" is highlighted by a metaphorical gesture of a stop-sign held up towards the projection, and again it is swept across the visual space facing the projection on the wall (line 8). This claim is however slightly nuanced by Axel, by his adding that "but maybe they happen to spill" (line 9). The reasoning about this continues as Elias furthers the argument that if it is spun "some fast, then it can spill some", and Axel seems to agree with this (line 10). Lucas, who has been silent during the other two boys' discussion, then verbalizes his experiences, and says that he has spun the bucket "really fast" and "all was kept". To explain this, Lucas uses his body, in catapulting a rapid spinning motion with his hand. He then follows to couple the word "kept" with a metaphorical gesture where he closes his hand around his fingers that are pinched together (line 11).

In this example, the scene is set by Axel who uses a deictic gesture to direct the attention to the screen. His iconical, sweeping gesture, is not coupled with his words, but the sentence is completed by the gesture, as if recalling or mirroring the spinning made outside that now is projected at the screen.

Elias seems to be uncertain about Axel's experience of water staying in the bucket while spinning. When Elias questions Axel about this he uses two main gestural components, accentuating the 'out' with a finger dropping down, as a metaphor, and a rise in intonation. The word "spinning", as commonly seen, is complemented by a spinning hand.

When Axel answers, it is coupled with a metaphorical gesture of the hand as the iconical stop-sign. Axel again uses the visual space in front of the boys, facing the screen, when quite slowly moving his hand over the projected picture as he says 'put', and prolongs the word to go with the gesture.

The slow movement over the projected space could be interpreted as adding weight to the argument. However, Axel adds some reservation to this claim. Elias suggests that if spun "some fast", water may run out, and this is agreed upon by Axel.

Lucas adds his experience to this conversation. When describing that he spun the bucket 'really fast', he uses the whole body in motion to launch the rapid spinning gesture of his hand. This can be interpreted as even further accentuating that the speed of the bucket is key, because Lucas claims that all his water was kept, and to illustrate this he encloses his hand around his pinched-together fingers - again as a way of embolden the argument with a metaphorical gesture, this time to highlight that all of the water was kept in the bucket.

This example illustrates how children can reason about the spinning motion, by recalling their earlier experience of spinning their buckets of water outside. When trying to explicate their sometimes different, experiences of abstract phenomena the children use, together with verbal language, several non-verbal means of their bodies, most markedly gestures. Gestures are used to illustrate or metaphorically liken the experiences of spinning a bucket with water. Gestures are used in conjunction with words, and other bodily 


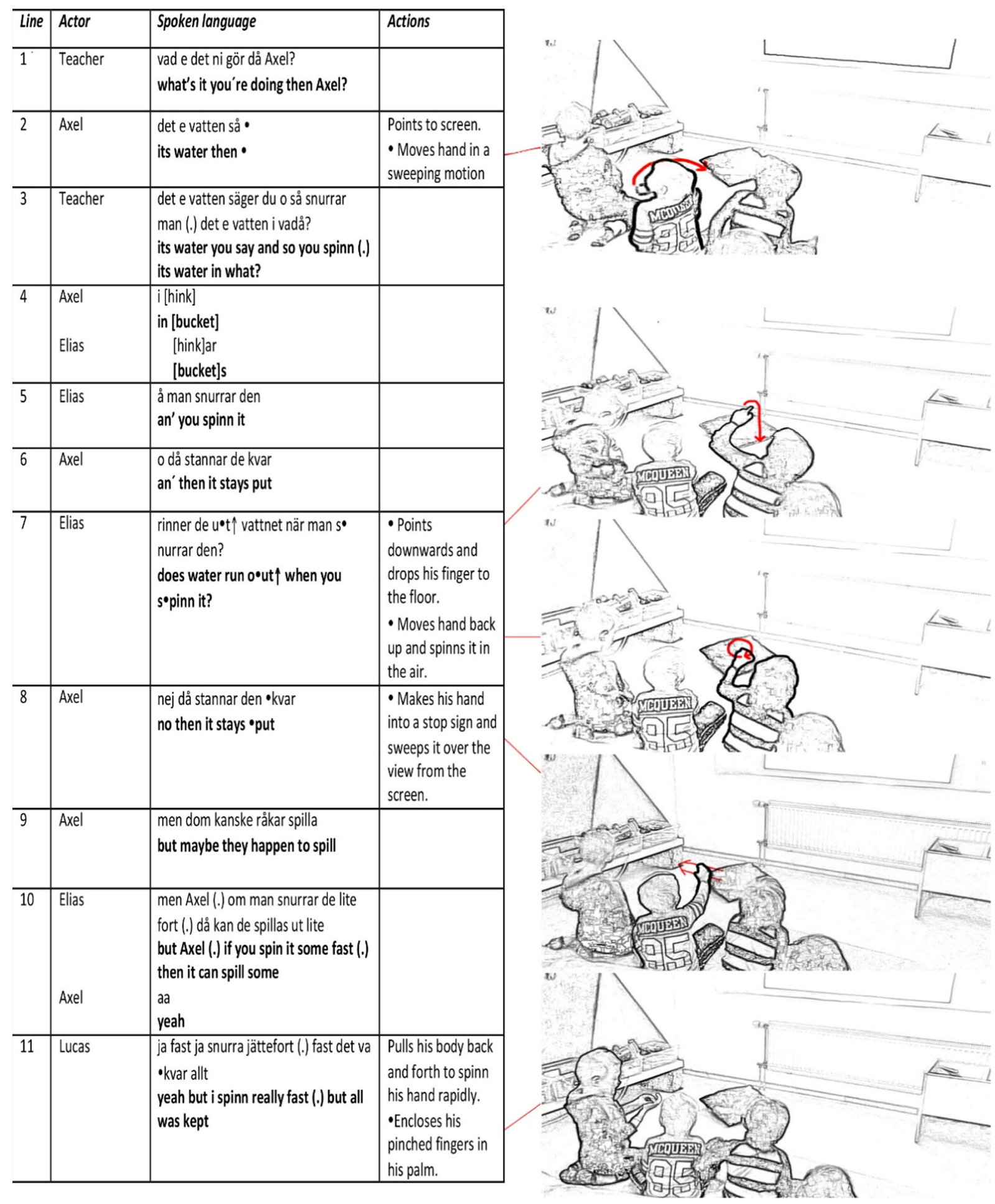

Excerpt 2. Children reasoning about spinning with their teacher in front of the screen.

means, in this case movement and pitch of voice. As such, the children can be said to reason with their bodies as an integral tool in their explanations. The experiences are thereby multimodally explicated in the intersection of bodily action and verbal argument. These arguments are also coupled with the surrounding and its specific artifacts, most notably in this case, with the surface of a projected digital image that is used to recall experiences but also as a deictically available auxiliary means for reasoning (c.f. Conezio \& French, 2002; Schoultz, Säljö \& Wyndhamn, 2001; Vygotsky, 1978). 


\section{Discussion}

This study has mapped how a group of children explores the concept of spinning in the institution of a Swedish preschool through mediating tools and artifacts, most notably the spinning top artifact. The children draw on different means, ranging from the perceptual to the verbal, when constructing, play with, and reason about the concept. This aligns with Pramling \& Pramling Samuelsson's (2001) observations of how children play with and test out concepts that are not completely grasped through artifacts. This study has closely analyzed the means children employ when they do this.

The preschool and its teachers use the children's interest in the spinning top toy, which can be seen as the central artifact (Vygotsky, 1978) of this study. They arrange the preschool environment, or Activity system (cf. Sannino, Daniels \& Gutiérrez, 2009) where tools and artifacts are arranged by the teachers in alignment with the object of the curricular science goals of preparatory physics education. With this, the teachers can configure (cf. Norris, 2014) environments and activities within them, where certain means may be more foregrounded to be in line with the pedagogical aims of the institution (cf. Hedegaard, 2007). Examples include adding more material for play with artifacts, or arranging activities that might enable children to experience the concept in new ways, as when children's fascination with spinning themselves is remodeled to a pedagogical activity in LA3. These observations follow well with the analysis of Klaar \& Öhman (2014) where preschool children explore concepts with their actions and how teachers' promotion of actions can enable further learning opportunities. This is also somewhat valid in Larsson's (2013) results. At the same time, it diverges somehow from Larsson's (2013) observation that teachers fail to observe children's learning opportunities. This study's results rather suggest how teachers can create learning activities both actively, through arranging pedagogical activities, and passively, by changing the environment and the artifacts in it. This should also be contrasted with the study by Sundberg et al. (2016) where a project about spinning rested on the teachers supplying artifacts for the children to explore by themselves. In the present study, children similarly explore the concepts through the artifacts of the preschool. However, the results also suggest how such external artifacts and their setting can be dynamically rearranged over time in a project. Experiences of such learning activities may be valuable in later guided activities, as seen in LA1, where new pieces enable new constructions and hence new "critical moments" in Larsson's (2013) terms. What can be seen as solitary construction and use of artifacts is, in this project, are later used as means in peer play (see LA2) and in reflective teacher-led activities (see LA4). Following this result, it is suggested that activities related to this theme should be seen within the wider context of the activity system (cf. Cole \& Engeström, 1993) and how it unfolds over time as children's conceptual understanding develops.

Children are frequently seen playing in the arranged environment, exemplified in multiple ways in LS 1, 2 and 3, where an array of means such as bodily movement and the use of hands, are used to explore the environment. For Ingold (2000) and Streeck (2009), this can be seen as ways of perceiving the environment, while at the same time also acting in it. Thus, it follows with Roth \& Lawless (2002, p. 380) that hands-on learning can promote new learning opportunities, and when teachers notice these they can be used for pedagogical goals as in LA3 and to enable talk comparable to the students in Roth \& Lawless's (2002) study as was exemplified in LA4. As Hedegaard (2007) and Nelson (2007) point out, preschools settings can be socioculturally arranged so that concepts may be explored in them, and the learning activities exemplifies how this might be done across a preschool science project merging children's tactile and everyday understanding with the preschools goals of abstract knowledge development.

In this project there have been two interacting goals of actors, or objects of Activity systems, sometimes with tensions (cf. Cole \& Engeström, 1993). One is the activity system of the preschool, with teachers working towards the object of the preschools curricular goals. Then there are the activity systems of the children, that might be both converging and diverging with the preschools', creating both tensions and possibilities as the children are both interested in the spinning phenomena, but also the Beyblade as an artifact of popular culture. However, in acting according to their interests, the children might simultaneously learn from the participation if the settings that are arranged by the preschool for learning experiences (cf. Nelson, 2007), as seen within the learning activities of this paper.

In this study, children have been shown to use language, sensory and other bodily means throughout the project observed. They use an array of different bodily means, such as gesture, touch, hearing, movement, gaze, and pitch of voice etc. Much of this interaction is embedded with the environment and its artifacts (cf. Goodwin, 2007), notably in ways that the spinning artifacts function as an object that children and teachers orient themselves towards. Language is embedded with children's explorations of these artifacts, and teachers may interact with children to enable new experiences and have children hypothesize and reason about these. This showcases the relation between everyday experience and the forming of conceptual understanding. Language is throughout the project coupled with other resources, and it can be suggested with Goldin-Meadow (2015) that there is scaffolding potential in a responsiveness to children's bodily and gestural expressive means, as this can be said to be an important source of ideas in development of the project during the weeks it was observed. In this way, this study also pinpoints the importance of understanding bodily means within sociocultural studies. They are shown to be a mode for communication for the preschool child, as much as an artifact or tool mediating between the child and the world. This study may expansively show what might be understood as auxiliary means in children's understanding and reasoning, following the tradition after Vygotsky (1978, 1986). It has also in these ways highlighted the dynamical relation between everyday and scientific concepts as foresighted by Vygotsky (1986), and illustrated some examples of how this might function in contemporary preschool activities.

\section{Conclusions}

This study has followed a group of preschool children as they, in a range of learning activities, explore a concept over 7 weeks. Following Conezio \& French (2002) who point to the role of science activities in preschool children's language learning, this study can 
at least illustrate how language learning and perception of concepts are intertwined in preschool activities, together with the scaffolding potential of artifacts. With Roth \& Lawless (2002) the study points to a movement towards increasingly verbally explicit explanations unfolding as the project develops, while at the same time, these explanations are seen to be coupled with other means in children's exploration.

It could be argued that this is the place where the dynamic between "spontaneous" and 'scientific' concepts (Vygotsky, 1986) is in action. This is shown to be harnessed by the teachers in this project, who rearrange the settings artifacts and employ activities to work with the ever-changing needs of the children. What this study may suggest, is that a closer look at what accounts for knowledge display for preschool children that includes all sorts of means of expression, may in turn be of use for teachers in promoting new learning opportunities. This also calls for more research exploring different mediational means in preschool children's conceptual development, and the ways in which activities and artifacts are worked as fundamental resources for children's present and potential ways of understanding the world around them.

\section{References}

Cole, M. (1996). Cultural psychology: a once and future discipline. Cambridge, Mass.: Belknap Press of Harvard University Press.

Cole, M., \& Engeström, Y. (1993). A cultural-historical approach to distributed cognition. In G. Salomon (Ed.). Distributed cognitions: Psychological and educational considerations (pp. 1-46). New York: Cambridge University Press.

Conezio, K., \& French, L. (2002). Science in the preschool classroom: Capitalizing on children's fascination with the everyday world to foster language and literacy development. Young Children (pp. 12-18). .

Engeström, Y. (1987). Learning by expanding: an activity-theoretical approach to developmental research. Univ. Helsinki.

Finnegan, R. H. (2014). Communicating: the multiple modes of human communication (2. ed.). London: Routledge.

Goldin-Meadow, S. (2015). From action to abstraction: Gesture as a mechanism of change. Developmental Review, 38, 167-184. http://dx.doi.org/10.1016/j.dr.2015. 07.007.

Goodwin, C. (1995). Seeing in Depth. Social Studies of Science, 25(2), 237-274. http://dx.doi.org/10.1177/030631295025002002.

Goodwin, C. (2007). Environmentally coupled gestures. In S. Duncan, J. Cassell, \& E. Levy (Eds.). Gesture and the dynamic dimensions of language (pp. 195-212). Amsterdam/Philadelphia: John Benjamins.

Hedegaard, M. (2007). The development of children's conceptual relation to the world, with focus on concept formation in preschool children's activity. In H. Daniels, M. Cole, \& J. Wertsch (Eds.). The Cambridge companion to Vygotsky. Cambridge University Press.

Ingold, T. (2000). The perception of the environment: Essays on livelihood, dwelling and skill. London: Routledge.

Klaar, S., \& Öhman, J. (2014). Doing, knowing, caring and feeling: Exploring relations between nature-oriented teaching and preschool children's learning. International Journal of Early Years Education, 22(1), 37-58. http://dx.doi.org/10.1080/09669760.2013.809655.

Knoblauch, H. (2005). Focused ethnography. Forum Qualitative Sozialforschung/Forum: Qualitative Social Research. 6(3).

Larsson, J. (2013). Children's encounters with friction. Friction understood as a phenomenon of emerging science and as 'opportunities for learning. Journal of Research in Childhood Education, 27(3), 377-392. http://dx.doi.org/10.1080/02568543.2013.796335.

McNeill, D. (1992). Hand and mind: What gestures reveal about thought. Chicago: Univ. of Chicago Press.

Nelson, K. (2007). Young minds in social worlds: experience, meaning, and memory. Cambridge, Mass: Harvard University Press.

Norris, S. (2014). Modal density and modal configurations. In C. Jewitt (Ed.). The Routledge handbook of multimodal analysis(Second edition). London. New York: Routledge.

Pink, S. (2015). Doing sensory ethnography (2. ed.). London: Sage.

Pramling, N., \& Pramling Samuelsson, I. (2001). "It's floating 'cause there is a hole." A young child's first experience of natural science. Early years. 21(2)http://dx.doi. org $/ 10.1080 / 713667696$

Roth, W.-M., \& Lawless, D. (2002). Science, culture, and the emergence of language. Science Education, 86, 368-385. http://dx.doi.org/10.1002/sce.10008.

Sannino, A., Daniels, H., \& Gutiérrez, K. D. (2009). Activity theory between historical engagement and future-making practice. In A. Sannino, H. Daniels, \& K. D. Gutiérrez (Eds.). Learning and expanding with activity theory (pp. 1-15). Cambridge: Cambridge University Press.

Schoultz, J., Säljö, R., \& Wyndhamn, J. (2001). Heavenly talk: Discourse, artifacts, and children's understanding of elementary astronomy. Human Development, 44, 103-118. http://dx.doi.org/10.1159/000057050.

Siraj-Blatchford, J. (2001). Emergent science and technology in the early years. XXIII World Congress of OMEP. Chile: Santiago.

Streeck, J. (2009). Gesturecraft: The manufacture of meaning. Amsterdam: John Benjamins Pub. Co.

Sundberg, B., Areljung, S., Due, K., Ekström, K., Ottander, C., \& Tellgren, B. (2016). Understanding preschool emergent science in a cultural historical context through Activity Theory. European Early Childhood Education Research Journal, 24(4), 567-580. http://dx.doi.org/10.1080/1350293X.2014.978557.

Thulin, S., \& Pramling, N. (2009). Anthropomorphically speaking: On communication between teachers and children in early childhood biology education. International Journal of Early Years Education, 17(2), 137-150. http://dx.doi.org/10.1080/09669760902982331.

Vygotsky, L. S. (1978). Mind in society: The development of higher psychological processes. Cambridge, Mass.: Harvard U.P.

Vygotsky, L. S. (1986). Thought and language (Rev. ed.). Cambridge, Mass.: MIT Press.

Wartofsky, M. (1979). Perception, representation, and the forms of action: Towards an historical epistemology. In R. S. C., \& M. Wartofsky (Eds.). Models: Representation and the scientific understanding (pp. 188-210). Springer Science and Business Media: Dordrecht, Holland. 